\title{
High Pass Filter and Bandpass Filter Using Current Feedback Operational Amplifier (CFOA)
}

\author{
Ridouane Hamdaouy ${ }^{\# 1^{*}}$, Khadija Slaoui ${ }^{\# 2}$ \\ \# University Sidi Mohamed Ben Abdellah, LESSI Laboratory, \\ Department of Physics Faculty of Sciences, \\ Dhar El Mehrez B.P. 1796, 30003 Fez-Atlas,Morocco
}

\begin{abstract}
The filter proposed in this paper has an advantage of higher the frequency response and better selectivity against the classical band pass filter.A active circuit current feedback operational amplifier (CFOA) has been designed and it uses the application of the implementing active filters, oscillators, rectifiers, and signal processing circuits. We observe that several active circuits have been proposed in the literature. In this study, we have proposed four inductance simulators that employ only one active circuit current feedback operational amplifier and three or four passive components. The first and fourth topologies are designed for series lossy inductance, whereas the second and third topologies are designed for lossless negative inductance simulators. A passive RLC filter is used to demonstrate the effectiveness of the proposed inductance simulators. The circuit design is synthesized using $130 \mathrm{~nm}$ CMOS technology with $\pm 3.3 \mathrm{~V}$ power supply in cadence virtuoso and the simulation is done using Spectre and the results agree with the theoretical analysis.
\end{abstract}

Keywords: CFOA, filter, CMOS

\section{INTRODUCTION}

Inductance is the source of many problems in electronic circuits and systems. It stands to reason that inductance radiates magnetic energy, it places a larger footprint in the integrated circuit, and it contains more parasitic noises than other components. Bulky and expensive passive inductors motivated the researchers to design the alternative circuits can be worked as inductors. Inductance simulators are widely used, especially for high frequencies, instead of inductors. Therefore, for designing filters or oscillator, for eliminating electromagnetic interferences the inductance simulators are used.

Recently, a considerable literature has grown up the theme of active inductance realization. Several active inductance simulators have been proposed such as operational transconductance amplifier (OTA) [1-3], operational transresistance amplifier (OTRA) [4,5], current-feedback operational amplifier (CFOA) [6-12] current differencing buffer amplifer (CDBA) [13,14], four terminal floating nullor (FTFN) [15], voltage differencing buffer amplifer (VDBA) [16-18], differential voltage current conveyor

$$
\left[\begin{array}{l}
I_{y} \\
I_{z} \\
V_{x} \\
V_{w}
\end{array}\right]=\left[\begin{array}{ccc}
0 & 0 & 0 \\
\alpha & 0 & 0 \\
0 & \beta_{1} & 0 \\
0 & 0 & \beta_{2}
\end{array}\right]\left[\begin{array}{l}
I_{x} \\
V_{y} \\
V_{z}
\end{array}\right]
$$

(DVCC) [19], second generation current conveyor (CCII) [20,21], dual-X current conveyor (DXCCII) [22-24]. Most of the reported circuits are commercially unavailable such as OTRA, CDBA, DVCC, DXCCII. Some of them such as FTFN [15] can be realized using two active devices such as AD844 CFOA can be commercially available. CFOA is a low-cost, general purpose device that has good AC and DC performance. CFOA is current mode circuit so it has some inherently advantages over the voltage mode operational amplifers such as wider bandwidth, wider dynamic range and greater linearity. It also allows high slew rate capability and it is free from the slew rate boundries that are basic characteristics of the traditional operational amplifiers.

Four different inductance simulators employing a single CFOA and three or four passive components were presented in [6]. Three different generic structures were also presented in [11] which employing a single CFOA and three or four passive components. The circuits [25-27] are not operated commercially available devices such as AD844, LM741. The circuits [4, 28-31] can be constructed with more than one AD844.

The overall structure of the study takes the form of four sections. The first section is an introduction, the second section gives the proposed four grounded inductance simulator topologies and parallel resonant circuit is constructed with the proposed inductance simulator, the third section gives the simulation results and the last section is the conclusion. It is expected that the proposed circuit will provide different opportunities to the designers accomplishing of analog integrated circuit ap plications.

\section{THE PROPOSED INDUCTOR SIMULATORS}

The equivalent circuit of CFOA is shown in Figure 1.a-b. In the ideal case, current gain and voltage gains are $\alpha=1$ and $\beta_{1}=\beta_{2}=1$ respectively. So; CFOA whose electrical symbol ideally specified as $\mathrm{I}_{\mathrm{y}}=0, \mathrm{I}_{\mathrm{x}}=\mathrm{I}_{\mathrm{z}}, \mathrm{V}_{\mathrm{x}}=\mathrm{V}_{\mathrm{y}}$, and $\mathrm{V}_{\mathrm{w}}=\mathrm{V}_{\mathrm{z}}$, are going to be stated by the following equation: 


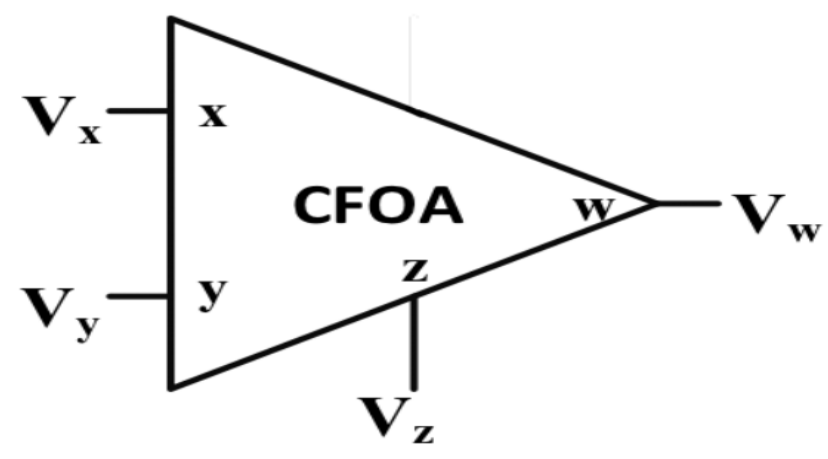

Figure 1.a.Equivalant circuit of CFOA

The simplified schematic of the Proposed Inductor Simulators discussed in this paper is shown in Fig. 1.b. It is made up of two voltage buffers, one at the input (transistors M1-M20) and the other at the output (transistors M1b-M20b). These buffers are realized from a well-known class AB differential stage [32] that is used here in unity gain. Transistors M16-M17 implement two level shifters allowing the analog ground to be set to $0 \mathrm{~V}$. The current at the inverting input terminal is mirrored to the highimpedance node at the intermediate stage (M21- M24) which provides the high transresistance gain. Voltage at node A is then buffered to the output by the second buffer. Dominant-pole frequency compensation is obtained through capacitor $C_{c}$ whereas resistor $R_{c}$ introduces, as usual, a negative zero.

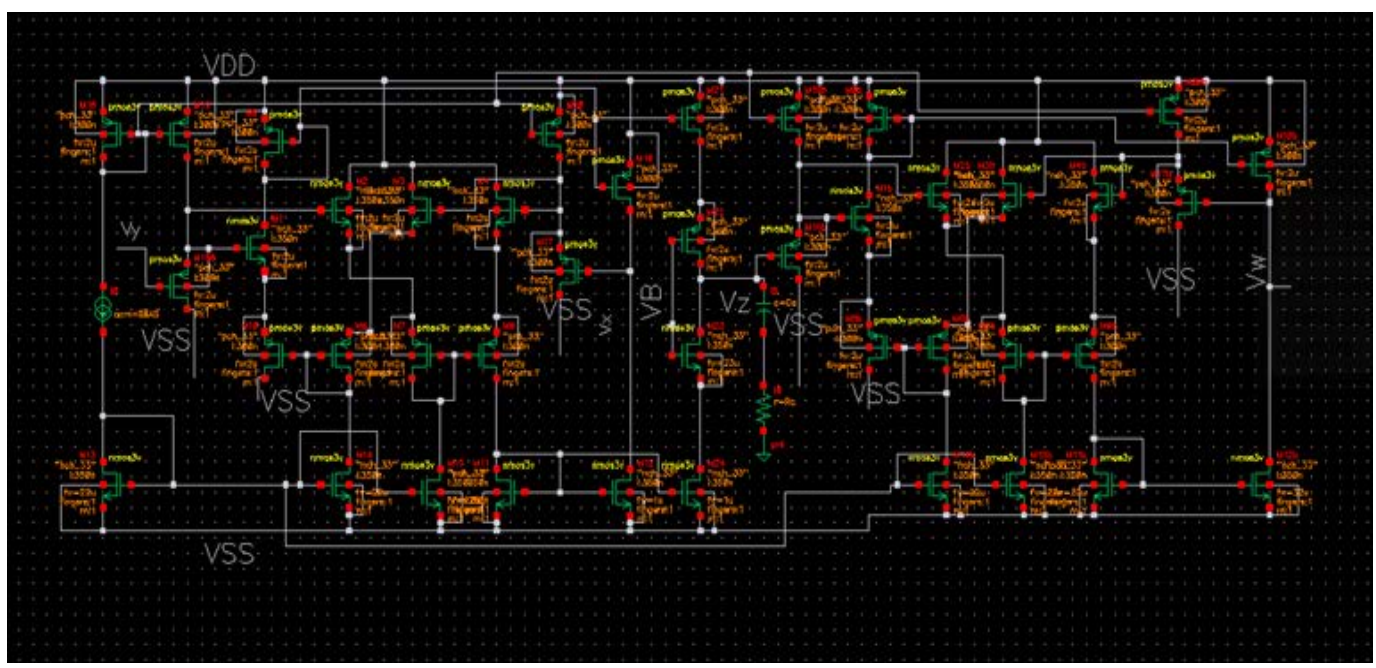

Fig. 1.b. Simplified schematic of the proposed current feedback operational amplifier.

Table 1.Equivalent impedances of proposed inductance simulators

\begin{tabular}{|l|c|c|}
\hline Circuit & Non-ideal impedances $\left(Z_{\text {eq }}\right)$ & Ideal impedances $\left(\mathrm{Z}_{\mathrm{eq}}\right)$ \\
\hline Figure 2 (a) & $\frac{R_{1}}{1+C s \alpha(\gamma-\beta) R_{2}}+\frac{R_{2}}{1+C s \alpha(\gamma-\beta) R_{2}}+\frac{R_{1} R_{2} C s \alpha \gamma}{1+C s \alpha(\gamma-\beta) R_{2}}$ & $R_{1} R_{2} C s+R_{1}+R_{2}$ \\
\hline Figure 2 (b) & $-\frac{R_{1} R_{2} C s}{\beta \gamma+C R_{2} \alpha(-1+\beta \gamma) R_{1}}$ & $-C R_{1} R_{2} s$ \\
\hline Figure 2 (c) & $\frac{R_{1} R_{2} C_{2} s}{R_{2} C_{2} s+\left(-\beta \gamma \alpha-\beta \gamma C_{1} R_{2} s \alpha\right)}$ & $\frac{R_{1} R_{2} C_{2} s}{R_{2} C_{2} s+\left(-1-C_{1} R_{2} s\right)}$ \\
\hline Figure 2 (d) & $\frac{R_{1}}{1-C s(-1+\beta \alpha \gamma) R_{2}}+\frac{R_{1} R_{2} C s}{1-C s(-1+\beta \alpha \gamma) R_{2}}$ & $R_{1} R_{2} C s+R_{1}$ \\
\hline
\end{tabular}


Table 2.Equivalent admittances of proposed inductance simulators ideal and non-ideal cases

\begin{tabular}{|l|l|c|}
\hline Circuit & Non-ideal admittances $\left(\mathrm{Y}_{\text {eq }}\right)$ & Ideal admittances $\left(Y_{\text {eq }}\right)$ \\
\hline Figure 2 (a) & $\frac{1}{R_{2}+R_{1}+C s \alpha \gamma R_{1} R_{2}}+\frac{C s \alpha(\gamma-\beta) R_{2}}{R_{2}+R_{1}+C s \alpha \gamma R_{1} R_{2}}$ & $\frac{1}{R_{1} R_{2} C s+R_{1}+R_{2}}$ \\
\hline Figure 2 (b) & $-\frac{\beta \gamma}{R_{1} R_{2} C s}+\frac{1-\beta \gamma}{R_{1}}$ & $\frac{1}{-C R_{1} R_{2} S}$ \\
\hline Figure 2 (c) & $\frac{1}{R_{1}}+\frac{C_{1} \alpha \gamma \beta}{R_{1} C_{2}}-\frac{\alpha \gamma \beta}{R_{1} R_{2} s C_{2}}$ & $\frac{1}{R_{1}}+\frac{C_{1}}{R_{1} C_{2}}-\frac{1}{R_{1} R_{2} s C_{2}}$ \\
\hline Figure 2 (d) & $\frac{1}{R_{1}+C s R_{1} R_{2}}+\frac{C s \alpha(1-\beta \alpha \gamma) R_{2}}{R_{1}+C s R_{1} R_{2}}$ & $\frac{1}{R_{1} R_{2} C s+R_{1}}$ \\
\hline
\end{tabular}

The proposed CFOA based inductor simulators are shown in Figure 2 a-d. The first inductance simulator consists of one CFOA and three passive components while the others consist of one CFOA and four passive components. Transfer functions of the proposed circuits are given in Table 1, 2. According to the equivalent impedance of the first and fourth simulators are intended for lossy series inductors. The second simulator is intended to negative lossless inductance simulator. The third one is also intended to negative lossless inductance simulator if $\mathrm{C}_{1}$ and $\mathrm{C}_{2}$ capacitors and $\mathrm{R}_{1}$ and $\mathrm{R}_{2}$ resistors are equal to each other.

$\mathbf{a}$
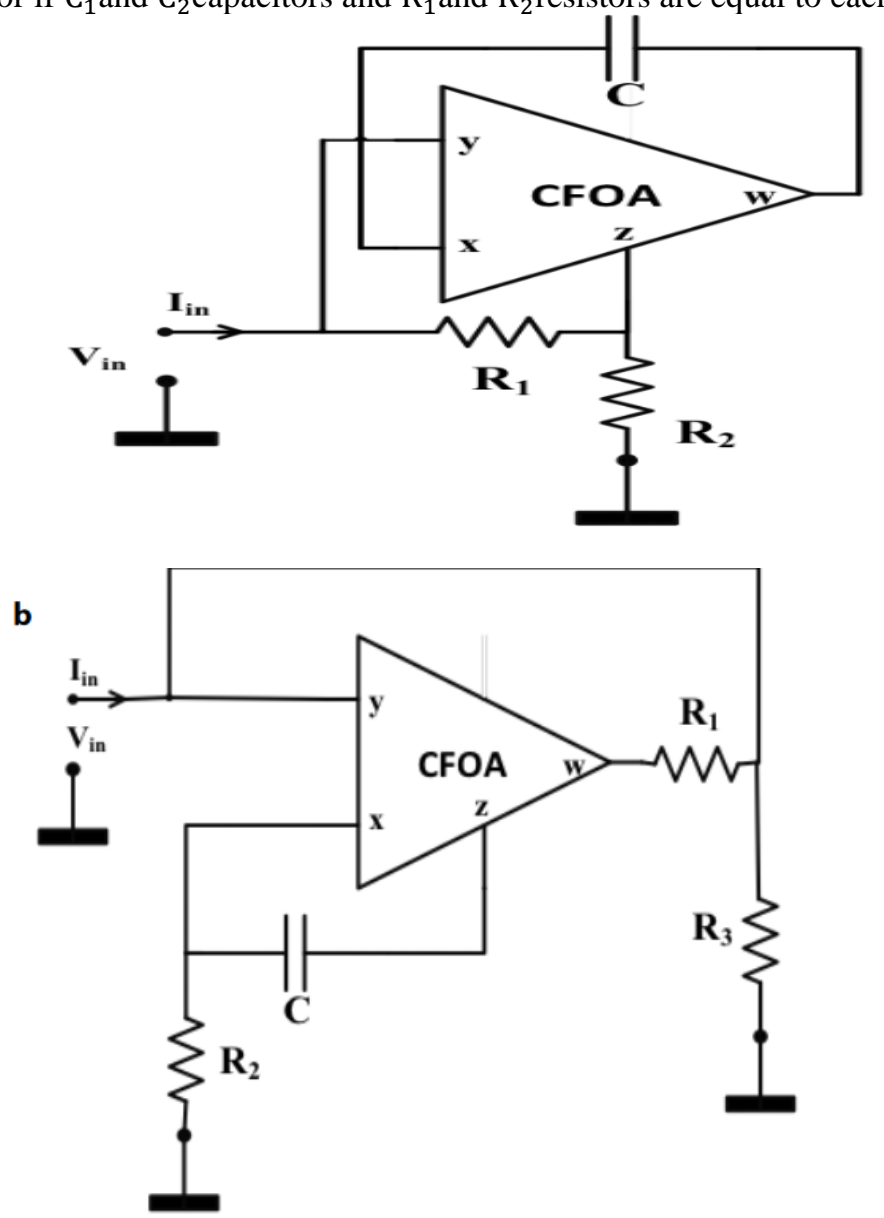

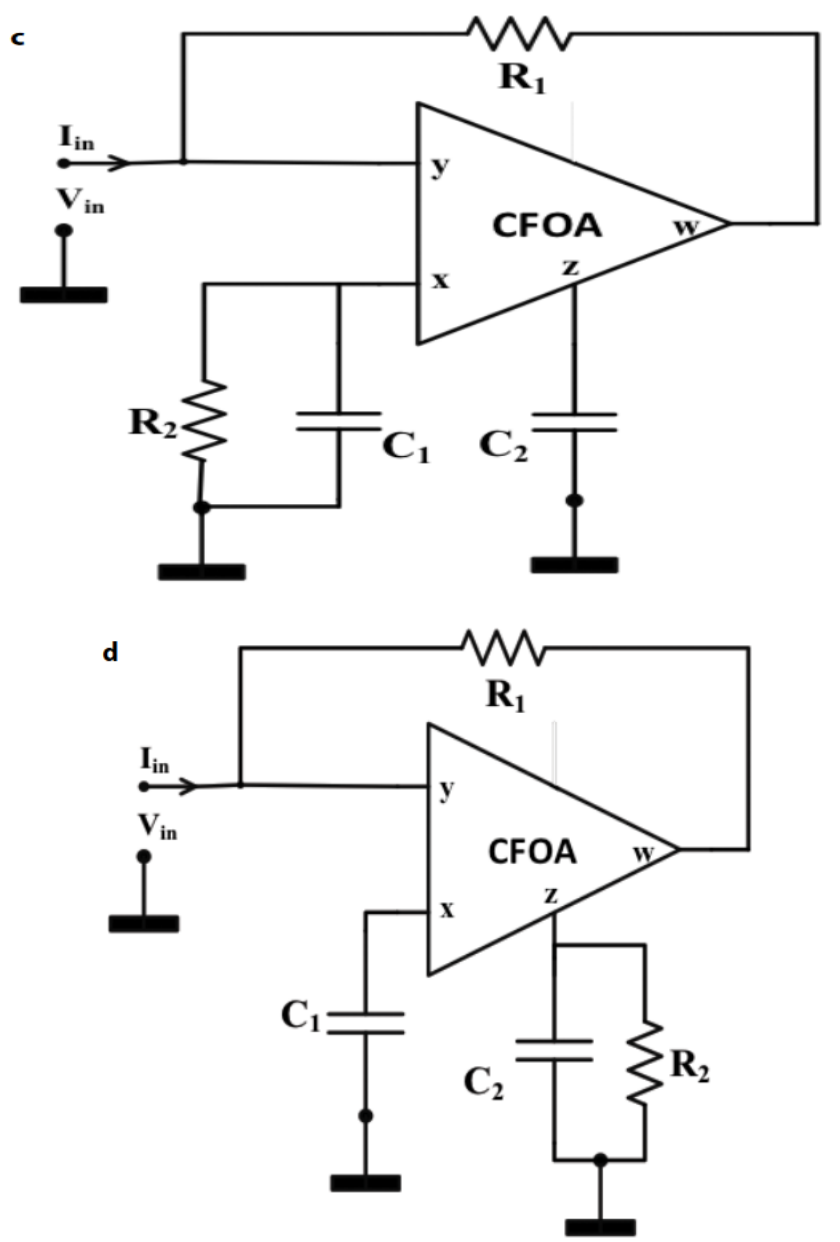

Figure 2. a-d.Proposed inductance simulators made with CFOA

\section{SIMULATION RESULTS}

As long as higher value inductances occupy a bigger area in chips, Inductor will be a central ingredient in deciding the total chip area because higher inductance values imply larger area consumption. In order to solve this problem, it is more convenient to use active implementations of an inductor which offer less area consumption.
TSMC13RF 0.13- $\mu \mathrm{m}$ CMOS technology is used to explain the performance of the presented inductance simulator. The AC response analysis is obtained to find the variation of magnitude Vs frequencies. The simulated frequency response of input impedance for the inductor simulator is given in Figure $2 \mathrm{~b}$. The magnitude of impedance of the presented inductance simulator is given in Figure 3. The inductive characteristic extends from $17.29 \mathrm{~Hz}$ to $73.16 \mathrm{MHz}$.

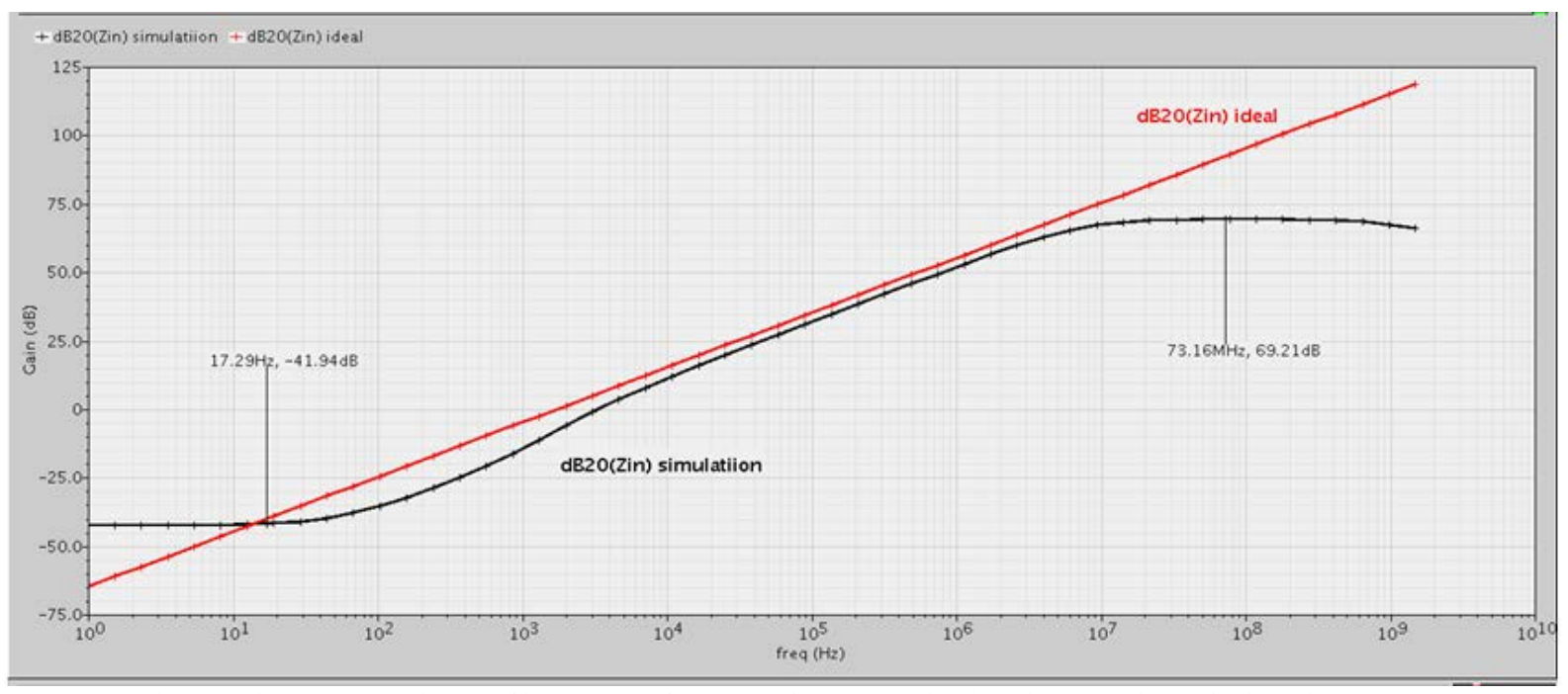

Figure 3.Simulated magnitudes of impedance of presented inductance simulator in comparison with ideal inductance 
RLC filter is presented as an application example to demonstrate the performance of the presented inductance simulator. Inductance simulator with a parallel capacitor and resistor formed as a resonant circuit shown in Figure 4. In this Figure actively simulated inductance simulator circuit in Figure $2 \mathrm{~b}$ replaces the parallel inductor.

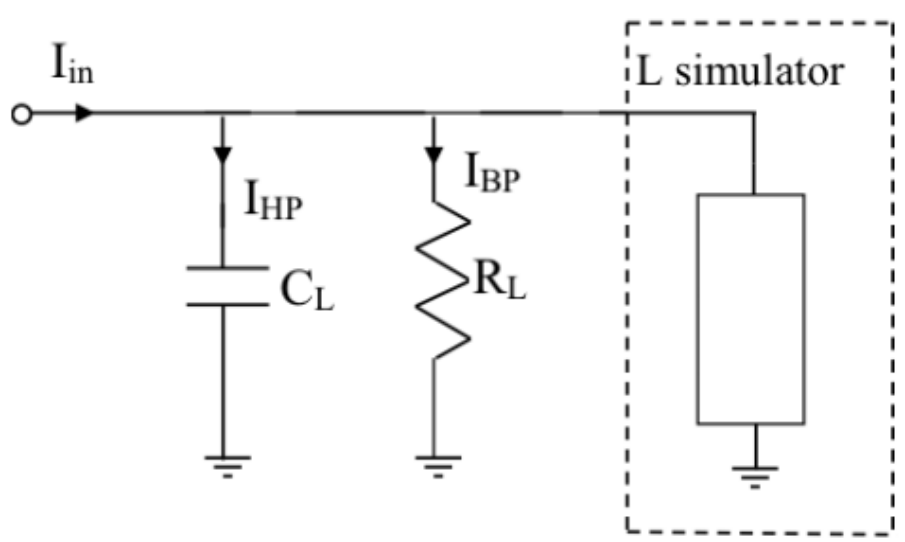

Figure 4.RLC Filter application of the proposed inductance simulator

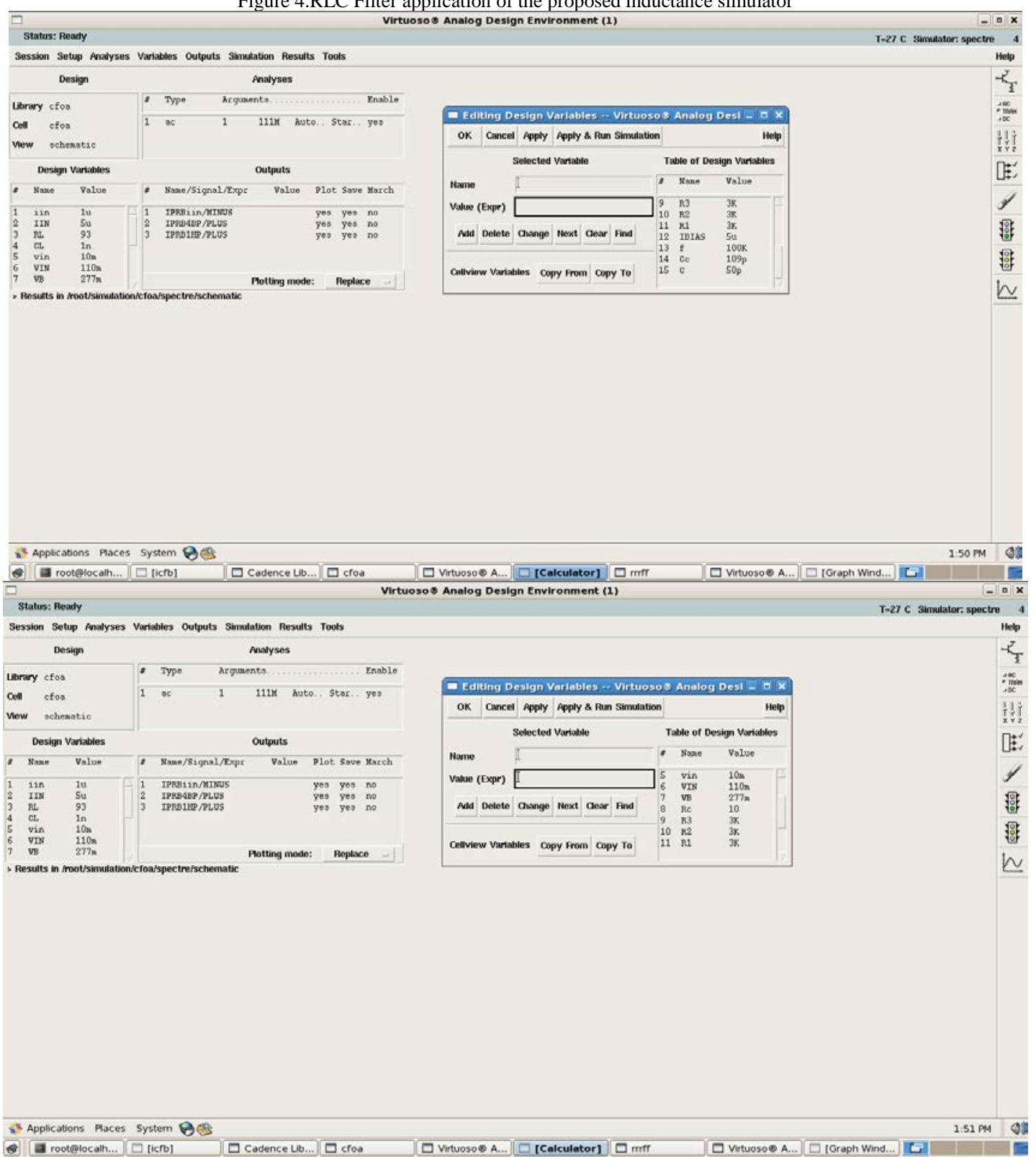


The CFOA is used to instrument a new Band pass filter and high pass filtre. The design makes use of a second-order filter built on a single CFOA [32]. By straight calculations the transfer functions are given by the following equations:

$$
\begin{aligned}
& \frac{I_{H P}(s)}{I_{\text {in }}(s)}=\frac{s^{2}}{S^{2}+s\left(\frac{1}{R_{L} C_{L}}\right)+\frac{1}{L_{e q} C_{L}}} \\
& \frac{I_{B P}(s)}{I_{\text {in }}(s)}=\frac{\left(\frac{1}{R_{L} C_{L}}\right) s}{S^{2}+s\left(\frac{1}{R_{L} C_{L}}\right)+\frac{1}{L_{e q} C_{L}}}
\end{aligned}
$$

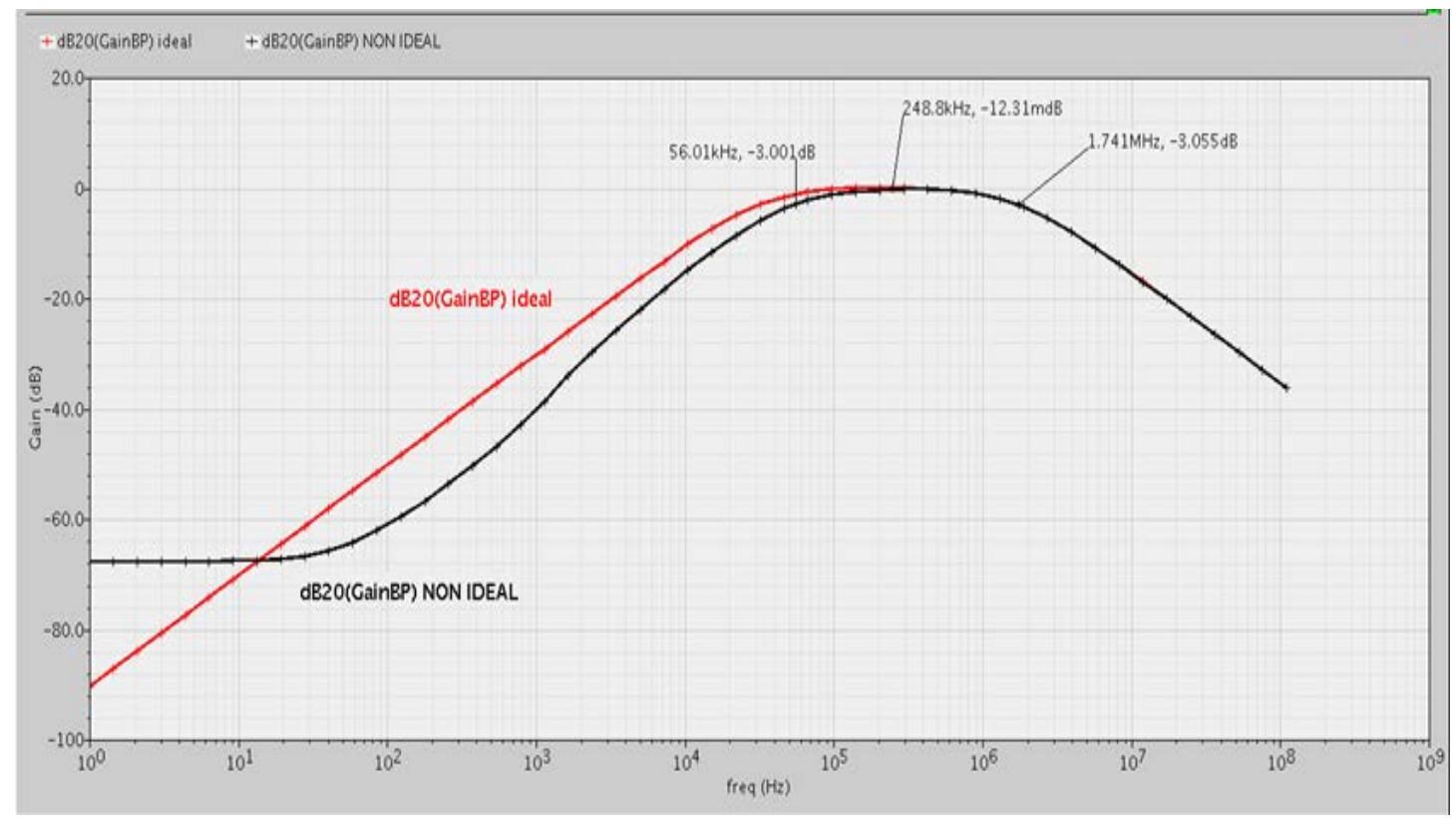

Figure 5.Simulated band pass response of the filter

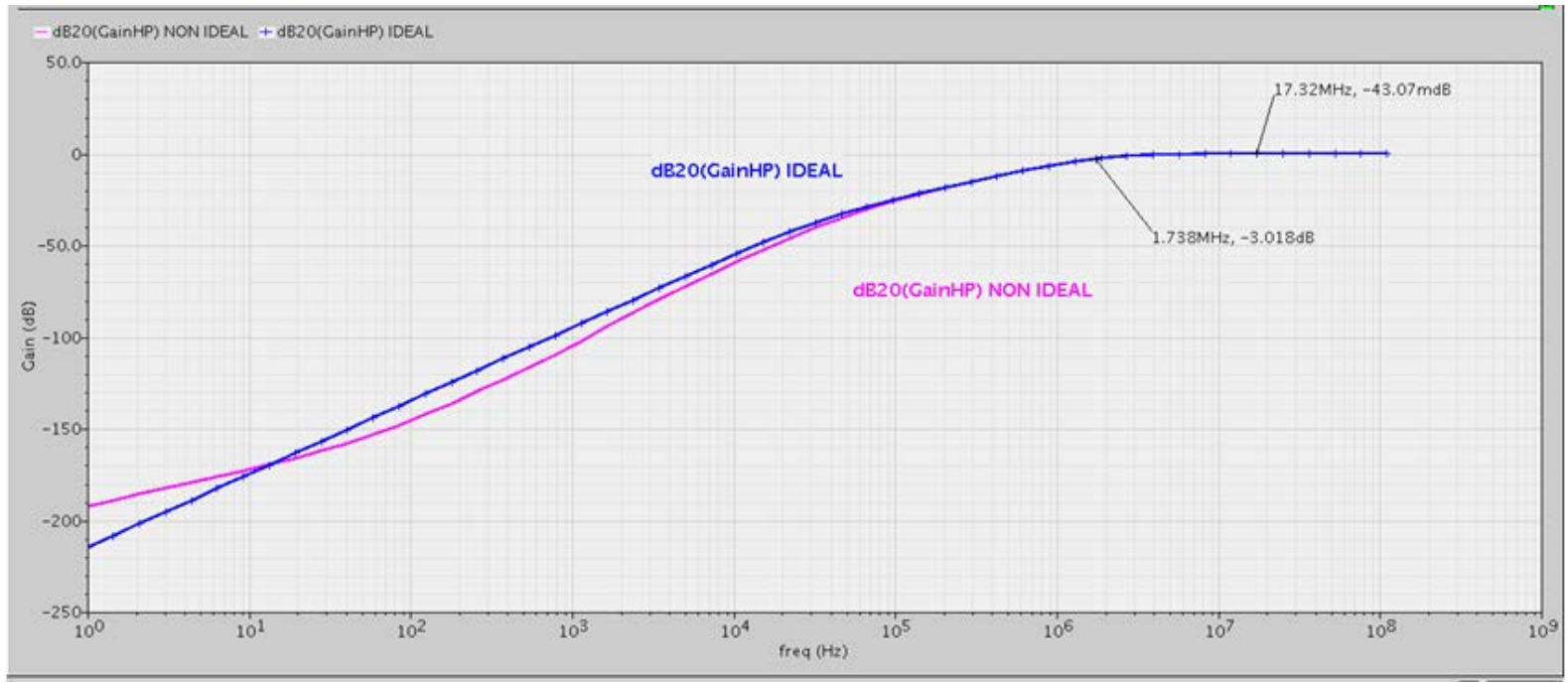

Figure 6.Simulated high pass response of the filter

The realized filter is simulated with CADENCE software in 0.13 um process using Simplified schematic of the proposed current feedback operational amplifier.

Supply voltages are taken as $\mathrm{V}_{\mathrm{DD}}=3.3 \mathrm{~V}$ and $\mathrm{V}_{\mathrm{SS}}=-3.3 \mathrm{~V}$. Simulation result of the filter responses, very good agreement with the predicted theory, is given in Figure 5 and Figure 6 respectively. The component values of the accomplished filter are chosen as follows: $C_{L}=1 n F, R_{L}=93 \Omega$
$\mathrm{R}_{1}=\mathrm{R}_{2}=\mathrm{R}_{3}=3 \mathrm{k} \Omega$ and $\mathrm{C}=50 \mathrm{pF}$, thus an inductor $\mathrm{L}_{\mathrm{eq}}=0.238 \mu \mathrm{H}$ is obtained. In order to analysis time responses of RLC filter, peak-to-peak $2 \mathrm{uA}$ and $100 \mathrm{KHz}$ sinusoidal inputs are applied.

The time domain analysis result is given in Figure 7 for bandpass and highpass filter configuration for the circuit in Figure 4. 


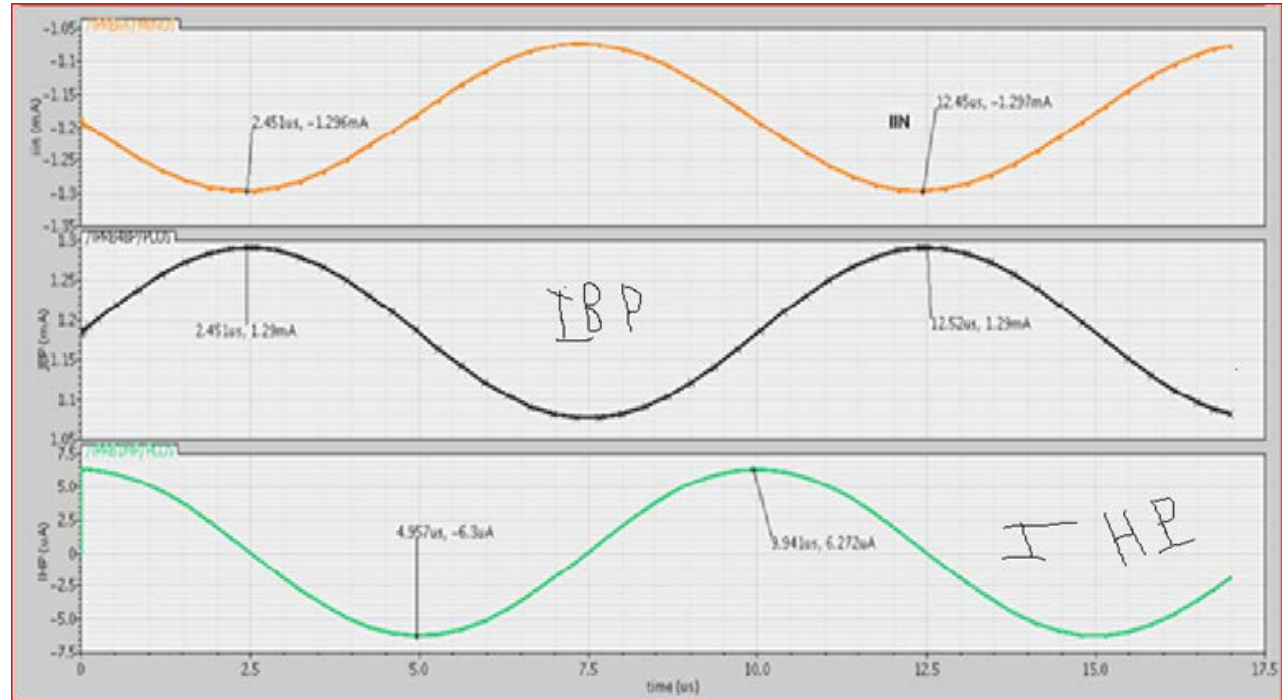

Figure 7.Time domain response of RLC filter high-pass and band- pass filter for 2uA peak-to-peak 100KHz sine wave input

\section{CONCLUSIONS}

This study has been presented an alternative configuration for the realization of a analysis responses of RLC filter. In this study, A CFOA based inductor simulators are proposed. The proposed circuit consisted only one single of CFOA, and three or four passive components. The aim of the present research was to propose the inductance simulators which consists three or four passive components in addition to single active device named CFOA. A CMOS CFOA has been designed, simulated and analysed using Cadence Tools. CFOA circuit has been applied in a complex pass filter with centre frequency f0. It is revealed that the gain can be programmed without altering the $\mathrm{Q}$ value of that filter.This circuit find more appropriate for varied range, low voltage and low power applications. The CFOA circuit is considered by the capacity to attain high Gain with low loss of bandwidth .

\section{REFERENCES}

[1] P. Soni, B. P. Singh, and M. Bhardwaj, "Design of OTA based floating inductor," in 2011 International Conference on Devices and Com-munications, ICDeCom 2011 - Proceedings, 2011. [CrossRef ]

[2] R. Banchuin, B. Chipipop, and B. Sirinaovakul, "Novel practically applicable passive equivalent circuit model of the alternatively structured higher performance practical OTA-based floating inductor,” in 2007 International Symposium on Intelligent Signal Processing and Communications Systems, ISPACS 2007 - Proceedings, pp. 447-450, 2008.

[3] R. Banchuin, R. Chaisricharoen, B. Chipipop, and B. Sirinaovakul, "In depth analysis of the CMOS OTA-based floating inductors," in 2006 International Symposium on Intelligent Signal Processing and Communications, ISPACS’06, pp. 239-242, 2007.

[4] U. Çam, F. Kaçar, O. Cicekoglu, H. Kuntman, and A. Kuntman, "Nov- el two OTRA-based grounded immitance simulator topologies,” Analog Integr. Circuits Signal Process., vol. 39, no. 2, pp. 169-175, 2004. [CrossRef ]

[5] B. C. Nagar, S. K. Paul, "Negative inductance simulator using OTRA,” vol. 3, no. 2, pp. 2-4, 2016. [CrossRef ]

[6] F. Kaçar and H. Kuntman, "CFOA-based lossless and lossy inductance simulators,” Radioengineering,vol. 20, no. 3, pp. 627-631, 2011.
[7] O. Çiçekoğlu, "Precise simulation of immittance functions using the CFOA,” MicroelectronicsJ.,vol. 29, no. 12, pp. 973-975, Dec. 1998. [CrossRef ]

[8] M. T. Abuelma'Atti, S. K. Dhar, “CFOA-based floating negative inductance, positive frequency dependent resistance and resis- tancecontrolled capacitance and resistance emulator,” Int. Conf. Electron. Information, Commun. ICEIC 2016, pp. 3-5, 2016.

[9] M. Taher, “A New CFOA-Based Low Frequency Lowpass Filter for Biomedical Applications,” pp. 1-4, 2016.

[10] M. Taher, "New CFOA-Based Floating Lossless Negative Immittance Function Emulators,” pp. 0-3, 2015.

[11] M. T. Abuelma'atti, "New grounded immittance function simulators using single current feedback operational amplifier," Analog Integr. Circuits Signal Process.,vol. 71, no. 1, pp. 95-100,2012. [CrossRef ]

[12] E. Yuce, "Novel lossless and lossy grounded inductor simulators consisting of a canonical number of components," Analog Integr. Circuits Signal Process.,vol. 59, pp. 77-82, 2009. [CrossRef ]

[13] J. K. Pathak, A. K. Singh, R. Senani, "New canonic lossy inductor using a single CDBA and its application,” Int. J. Electron., vol. 103, no. 1, pp. 1-13, 2016. [CrossRef ]

[14] S. Minaei, E. Yuce, "A simple CMOS-based inductor simulator and frequency performance improvement techniques,” AEU - Int. J. Electron. Commun.,vol. 66, no. 11, pp. 884-891, 2012. [CrossRef ]

[15] U. Çam, O. Ciçekoğlu, H. Kuntman, "Novel lossless floating immitance simulator employing only two FTFNs,” Analog Integr. Circuits Signal Process.,vol. 29, no. 3, pp. 233-235, 2001. [CrossRef ]

[16] O. Channumsin, J. Pimpol, C. Thongsopa, W. Tangsrirat, "VD-BAbased floating inductance simulator with a grounded ca-pacitor,” in Proceedings - 2015 7th International Conference on Information Technology and Electrical Engineering: Envisioning the Trend of Computer, Information and Engineering, ICITEE 2015, 2015, pp. 114-117. [CrossRef ]

[17] A. Yeşil, F. Kaçar, K. Gürkan, "Lossless grounded inductance simulator em-ploying single VDBA and its experimental band-pass filter application,” AEU - Int. J. Electron. Commun.,vol. 68,no. 2, pp. 143-150, 2014. [CrossRef ]

[18] A. Yesil and F. Kacar, "VDBA-based lossless and lossy inductance simula-tors and its filter applications," in 2016 24th Signal Processing and Com-munication Application Conference (SIU), 2016, pp. 909-912. [CrossRef]

[19] A. Abaci and E. Yuce, "Modified DVCC based quadrature oscillator and lossless grounded inductor simulator using grounded capacitor(s),” AEU - Int. J. Electron. Commun.,vol. 76, pp. 86-96, 2017. [CrossRef ]

[20] E. Yuce, O. Cicekoglu, S. Minaei, "CCII-based grounded to floating im- mittance converter and a floating inductance simulator,” Analog Inte-gr. Circuits Signal Process., vol. 46, no. 3, pp. 287291, 2006. [CrossRef ] 
[21] G. Ferri, N. Guerrini, E. Silverii, A. Tatone, "Vibration damping using CCII-based inductance simulators,” IEEE Trans. Instrum. Meas., vol. 57, no. 5, pp. 907-914, 2008. [CrossRef ]

[22] A. Yeşil and F. Kaçar, "New dxccii-based grounded series inductance simulator topologies," İstanbul Univ. - J. Electr. Electron. Eng.,vol. 14, no. 2, pp. 1785-1789, 2015.

[23] I. Myderrizi, S. Minaei, E. Yuce, "DXCCII-based grounded inductance simulators and filter applications,” Microelectronics J.,vol. 42, no. 9, pp. 1074-1081, 2011. [CrossRef ]

[24] B. Metin, "Supplementary inductance simulator topologies employing single DXCCII,” Radioengineering,vol. 20, no. 3, pp. 614618, 2011.

[25] M. Incekaraoglu and U. Çam, "Realization of Series and Parallel RL and C-D Impedances Using Single Differential Voltage Current Conveyor,” Analog Integr. Circuits Signal Process.,vol. 43,no. 1, pp. 101-104, Apr. 2005. [CrossRef ]

[26] E. Yuce, "Comment on 'realization of series and parallel R-L and CD im-pedances using single differential voltage current conveyor,’” Analog Integr. Circuits Signal Process.,vol. 49, no. 1, pp. 91-92, 2006. [CrossRef ]

[27] H. Y. Wang and C.T. Lee, "Systematic synthesis of R-L and C-D im-mittances using single CCIII," Int. J. Electron., vol. 87, no. December 2011, pp. 293-301, 2000.

[28 ]H. Y. Wang and C. T. Lee, "Realisation of R-L and C-D immittances us-ing single FTFN,” Electron. Lett., vol. 34, no. 6, p. 502, 1998. [CrossRef ]

[29] M. O. Cicekoglu, "Active simulation of grounded inductors with CCII's and grounded passive elements,” Int. J. Electron., vol. 85, no. 4, pp. 455-462, 1998. [CrossRef ]

[30] E. Yuce and S. Minaei, "A modified CFOA and its applications to simulated inductors, capacitance multipliers, and analog filters,” IEEE Trans. Circuits Syst. I Regul. Pap.,vol. 55, no. 1, pp. 266-275, 2008. [CrossRef ]

[31] O. Çiçekolu, A. Toker, and H. Kuntman, "Universal immittance func-tion simulators using current conveyors," Comput. Electr. Eng.,vol. 27, no. 3, pp. 227-238, 2001. [CrossRef ]

[32] P. W. Li, M. J. Chin, P. R. Gray, and R. Castello, "A ratioindependent algorithmic analog-to digital conversion technique,” IEEE J.Solid-State Circuits, vol. SC-19, pp. 828-836, Dec. 1984. 